\title{
AN EXAMPLE FOR A ONE-PARAMETER NONEXPANSIVE SEMIGROUP
}

TOMONARI SUZUKI

Received 20 December 2003

We give one example for a one-parameter nonexpansive semigroup. This example shows that there exists a one-parameter nonexpansive semigroup $\{T(t): t \geq 0\}$ on a closed convex subset $C$ of a Banach space $E$ such that $\lim _{t \rightarrow \infty}\left\|(1 / t) \int_{0}^{t} T(s) x d s-x\right\|=0$ for some $x \in C$, which is not a common fixed point of $\{T(t): t \geq 0\}$.

\section{Introduction}

Throughout this paper, we denote by $\mathbb{N}$ and $\mathbb{R}$ the sets of positive integers and real numbers, respectively.

A family $\{T(t): t \geq 0\}$ of mappings on $C$ is called a one-parameter nonexpansive semigroup on a subset $C$ of a Banach space $E$ if the following hold:

( $\operatorname{sg} 1)$ for each $t \geq 0, T(t)$ is a nonexpansive mapping on $C$, that is,

$$
\|T(t) x-T(t) y\| \leq\|x-y\| \quad \forall x, y \in C ;
$$

(sg2) $T(0) x=x$ for all $x \in C$;

(sg3) $T(s+t)=T(s) \circ T(t)$ for all $s, t \geq 0$;

(sg4) for each $x \in C$, the mapping $t \mapsto T(t) x$ is continuous.

We know that $\{T(t): t \geq 0\}$ has a common fixed point under the assumption that $C$ is weakly compact convex and $E$ has the Opial property; see $[3,4,5,6,8,10,12]$ and other works.

Convergence theorems for one-parameter nonexpansive semigroups are proved in $[1,2,9,11,13,15]$ and other works. For example, Baillon and Brezis in [2] proved the following theorem; see also [16, page 80$]$.

Theorem 1.1 (Baillon and Brezis [2]). Let $C$ be a bounded closed convex subset of a Hilbert space $E$ and let $\{T(t): t \geq 0\}$ be a one-parameter nonexpansive semigroup on $C$. Then, for any $x \in C$,

$$
\frac{1}{t} \int_{0}^{t} T(s) x d s
$$

converges weakly to a common fixed point of $\{T(t): t \geq 0\}$ as $t \rightarrow \infty$. 
Also, Suzuki and Takahashi in [15] proved the following.

Theorem 1.2 (Suzuki and Takahashi [15]). Let $C$ be a compact convex subset of a Banach space $E$ and let $\{T(t): t \geq 0\}$ be a one-parameter nonexpansive semigroup on $C$. Let $x_{1} \in C$ and define a sequence $\left\{x_{n}\right\}$ in $C$ by

$$
x_{n+1}=\frac{\alpha_{n}}{t_{n}} \int_{0}^{t_{n}} T(s) x_{n} d s+\left(1-\alpha_{n}\right) x_{n}
$$

for $n \in \mathbb{N}$, where $\left\{\alpha_{n}\right\} \subset[0,1]$ and $\left\{t_{n}\right\} \subset(0, \infty)$ satisfy the following conditions:

$$
0<\liminf _{n \rightarrow \infty} \alpha_{n} \leq \limsup _{n \rightarrow \infty} \alpha_{n}<1, \quad \lim _{n \rightarrow \infty} t_{n}=\infty, \quad \lim _{n \rightarrow \infty} \frac{t_{n+1}}{t_{n}}=1 .
$$

Then $\left\{x_{n}\right\}$ converges strongly to a common fixed point $z_{0}$ of $\{T(t): t \geq 0\}$.

The following theorem plays a very important role in the proof of Theorem 1.2.

Theorem 1.3 (Suzuki and Takahashi [15]). Let $C$ be a compact convex subset of a Banach space E. Let $\{T(t): t \geq 0\}$ be a one-parameter nonexpansive semigroup on $C$. Then for $z \in$ $C$, the following are equivalent:

(i) $z$ is a common fixed point of $\{T(t): t \geq 0\}$;

(ii)

$$
\liminf _{t \rightarrow \infty}\left\|\frac{1}{t} \int_{0}^{t} T(s) z d s-z\right\|=0
$$

holds.

Recently, Suzuki proved in [14] the following result similar to Theorem 1.3. This theorem also plays a very important role in the proof of the existence of some nonexpansive retraction onto the set of common fixed points.

Theorem 1.4 (Suzuki [14]). Let E be a Banach space with the Opial property and let $C$ be a weakly compact convex subset of $E$. Let $\{T(t): t \geq 0\}$ be a one-parameter nonexpansive semigroup on $C$. Then for $z \in C$, the following are equivalent:

(i) $z$ is a common fixed point of $\{T(t): t \geq 0\}$;

(ii) formula (1.5) holds;

(iii) there exists a subnet of a net

$$
\left\{\frac{1}{t} \int_{0}^{t} T(s) z d s\right\}
$$

in $C$ converging weakly to $z$.

So, it is a natural problem whether or not the conclusion of Theorems 1.3 and 1.4 holds in general. In this paper, we give one example concerning Theorems 1.3 and 1.4. This example shows that there exists a one-parameter nonexpansive semigroup $\{T(t): t \geq 0\}$ 
on a closed convex subset $C$ of a Banach space $E$ such that

$$
\lim _{t \rightarrow \infty}\left\|\frac{1}{t} \int_{0}^{t} T(s) x d s-x\right\|=0
$$

for some $x \in C$, which is not a common fixed point of $\{T(t): t \geq 0\}$. That is, our answer of the problem is negative.

\section{Example}

We give one example concerning Theorems 1.3 and 1.4. See also [7, Example 3.7].

Example 2.1. Put $\Omega=\{-1\} \cup[0, \infty)$, let $E$ be the Banach space consisting of all bounded continuous functions on $\Omega$ with supremum norm, and define a subset $C$ of $E$ by

$$
C=\left\{x \in E: \begin{array}{l}
0 \leq x(u) \leq 1 \text { for } u \in \Omega, \\
\left|x\left(u_{1}\right)-x\left(u_{2}\right)\right| \leq\left|u_{1}-u_{2}\right| \text { for } u_{1}, u_{2} \in[0, \infty)
\end{array}\right\} .
$$

Define a nonexpansive semigroup $\{T(t): t \geq 0\}$ as follows. For $t \in[0,1]$, define

$$
(T(t) x)(u)= \begin{cases}x(u), & \text { if } u=-1, \\ x(u-t), & \text { if } u \geq t, \\ x(0)-t+u, & \text { if } 0 \leq u \leq t, \\ & 1-\alpha_{x}(1-t+u) \leq x(0)-t+u, \\ x(0)+t-u, & \text { if } 0 \leq u \leq t, \\ & 1-\alpha_{x}(1-t+u) \geq x(0)+t-u, \\ 1-\alpha_{x}(1-t+u), & \text { if } 0 \leq u \leq t, \\ & \left|1-\alpha_{x}(1-t+u)-x(0)\right| \leq t-u,\end{cases}
$$

where

$$
\alpha_{x}(1-t+u)=\sup \{x(s): s \in\{-1\} \cup[1-t+u, \infty)\} .
$$

For $t \in(1, \infty)$, there exist $m \in \mathbb{N}$ and $t^{\prime} \in[0,1 / 2)$ satisfying $t=m / 2+t^{\prime}$. Define $T(t)$ by

$$
T(t)=T\left(\frac{1}{2}\right)^{m} \circ T\left(t^{\prime}\right)
$$

Then $0 \in C$ is not a common fixed point of $\{T(t): t \geq 0\}$ and

$$
\lim _{t \rightarrow \infty}\left\|\frac{1}{t} \int_{0}^{t} T(s) 0 d s-0\right\|=0
$$

holds.

Before proving Example 2.1, we need some lemmas. 
Lemma 2.2. The following hold:

(i) $\left|\alpha_{x}\left(u_{1}\right)-\alpha_{x}\left(u_{2}\right)\right| \leq\left|u_{1}-u_{2}\right|$ for $x \in C$ and $u_{1}, u_{2} \in[0, \infty)$;

(ii) $\left|\alpha_{x}(u)-\alpha_{y}(u)\right| \leq\|x-y\|$ for $x, y \in C$ and $u \in[0, \infty)$.

Proof. We first show (i). Without loss of generality, we may assume $u_{1}<u_{2}$. For $s \in$ $\left[u_{1}, u_{2}\right]$, we have $\left|x(s)-x\left(u_{2}\right)\right| \leq\left|s-u_{2}\right|$ and hence

$$
x(s) \leq x\left(u_{2}\right)+\left|s-u_{2}\right| \leq \alpha_{x}\left(u_{2}\right)+\left|u_{1}-u_{2}\right| .
$$

For $s \in\left[u_{2}, \infty\right)$, we have

$$
x(s) \leq \alpha_{x}\left(u_{2}\right) \leq \alpha_{x}\left(u_{2}\right)+\left|u_{1}-u_{2}\right| .
$$

Hence,

$$
\alpha_{x}\left(u_{1}\right) \leq \alpha_{x}\left(u_{2}\right)+\left|u_{1}-u_{2}\right|
$$

holds. Since $\alpha_{x}\left(u_{2}\right) \leq \alpha_{x}\left(u_{1}\right)$, we obtain

$$
\left|\alpha_{x}\left(u_{1}\right)-\alpha_{x}\left(u_{2}\right)\right| \leq\left|u_{1}-u_{2}\right| .
$$

We next show (ii). For each $\varepsilon>0$, there exists $s \in\{-1\} \cup[u, \infty)$ satisfying $x(s)>\alpha_{x}(u)-\varepsilon$. We have

$$
\alpha_{x}(u)-\alpha_{y}(u) \leq x(s)+\varepsilon-y(s) \leq\|x-y\|+\varepsilon .
$$

Since $\varepsilon$ is arbitrary, we have $\alpha_{x}(u)-\alpha_{y}(u) \leq\|x-y\|$. Similarly we obtain $\alpha_{y}(u)-\alpha_{x}(u) \leq$ $\|x-y\|$ and hence $\left|\alpha_{x}(u)-\alpha_{y}(u)\right| \leq\|x-y\|$.

Lemma 2.3. Fix $x \in C, t \in[0,1]$, and $u_{1}, u_{2}$ with $0 \leq u_{1} \leq u_{2} \leq t$. Then the following hold:

(i) $1-\alpha_{x}\left(1-t+u_{1}\right)<(T(t) x)\left(u_{2}\right)-u_{2}+u_{1}$ implies that $(T(t) x)\left(u_{1}\right)=x(0)-t+u_{1}$ and $(T(t) x)\left(u_{2}\right)=x(0)-t+u_{2}$;

(ii) $1-\alpha_{x}\left(1-t+u_{1}\right)>(T(t) x)\left(u_{2}\right)+u_{2}-u_{1}$ implies that $(T(t) x)\left(u_{1}\right)=x(0)+t-u_{1}$ and $(T(t) x)\left(u_{2}\right)=x(0)+t-u_{2}$;

(iii) $\left|1-\alpha_{x}\left(1-t+u_{1}\right)-(T(t) x)\left(u_{2}\right)\right| \leq u_{2}-u_{1}$ implies that $(T(t) x)\left(u_{1}\right)=1-\alpha_{x}(1-$ $\left.t+u_{1}\right)$.

Remark 2.4. One and only one of the assumptions (i), (ii), and (iii) holds.

Proof. We first prove (i). We assume that $1-\alpha_{x}\left(1-t+u_{2}\right)>x(0)-t+u_{2}$. Then by the definition of $T(t)$,

$$
(T(t) x)\left(u_{2}\right)=\min \left\{x(0)+t-u_{2}, 1-\alpha_{x}\left(1-t+u_{2}\right)\right\}
$$

So, we have

$$
(T(t) x)\left(u_{2}\right)-u_{2}+u_{1} \leq 1-\alpha_{x}\left(1-t+u_{2}\right)-u_{2}+u_{1} \leq 1-\alpha_{x}\left(1-t+u_{1}\right)
$$


by Lemma 2.2. This is a contradiction. Therefore we obtain $1-\alpha_{x}\left(1-t+u_{2}\right) \leq x(0)-$ $t+u_{2}$. Hence $(T(t) x)\left(u_{2}\right)=x(0)-t+u_{2}$. Since

$$
1-\alpha_{x}\left(1-t+u_{1}\right)<(T(t) x)\left(u_{2}\right)-u_{2}+u_{1}=x(0)-t+u_{1},
$$

we have $(T(t) x)\left(u_{1}\right)=x(0)-t+u_{1}$. Similarly, we can prove (ii). We finally prove (iii). We assume that $1-\alpha_{x}\left(1-t+u_{1}\right)<x(0)-t+u_{1}$. Then by Lemma 2.2 , we have

$$
\begin{aligned}
1-\alpha_{x}\left(1-t+u_{2}\right) & \leq 1-\alpha_{x}\left(1-t+u_{1}\right)+u_{2}-u_{1} \\
& <x(0)-t+u_{1}+u_{2}-u_{1}=x(0)-t+u_{2} .
\end{aligned}
$$

Hence $(T(t) x)\left(u_{2}\right)=x(0)-t+u_{2}$. So,

$$
(T(t) x)\left(u_{2}\right)-\left(1-\alpha_{x}\left(1-t+u_{1}\right)\right)>\left(x(0)-t+u_{2}\right)-\left(x(0)-t+u_{1}\right)=u_{2}-u_{1} .
$$

This is a contradiction. Therefore we obtain $1-\alpha_{x}\left(1-t+u_{1}\right) \geq x(0)-t+u_{1}$. Similarly we can prove that $1-\alpha_{x}\left(1-t+u_{1}\right) \leq x(0)+t-u_{1}$. Hence $(T(t) x)\left(u_{1}\right)=1-\alpha_{x}(1-t+$ $\left.u_{1}\right)$.

Proof of Example 2.1. It is clear that $C$ is closed and convex. We first prove that $T(t) x \in C$ for all $t \in[0,1]$ and $x \in C$. It is clear that

$$
\begin{aligned}
& 0 \leq(T(t) x)(-1)=x(-1) \leq 1, \\
& 0 \leq(T(t) x)(u)=x(u-t) \leq 1
\end{aligned}
$$

for $u \in[t, \infty)$. For $u \in[0, t]$, since $0 \leq 1-\alpha_{x}(1-t+u) \leq 1, x(0)-t+u \leq x(0) \leq 1$ and $x(0)+t-u \geq x(0) \geq 0$, we have $0 \leq(T(t) x)(u) \leq 1$. Fix $u_{1}, u_{2} \in[0, \infty)$ with $u_{1}<u_{2}$. In the case when $t \leq u_{1}$, we have

$$
\begin{aligned}
\left|(T(t) x)\left(u_{1}\right)-(T(t) x)\left(u_{2}\right)\right| & =\left|x\left(u_{1}-t\right)-x\left(u_{2}-t\right)\right| \\
& \leq\left|\left(u_{1}-t\right)-\left(u_{2}-t\right)\right|=\left|u_{1}-u_{2}\right| .
\end{aligned}
$$

In the case when $u_{2} \leq t$, by Lemma 2.3 , it is easily proved that $\left|(T(t) x)\left(u_{1}\right)-(T(t) x)\left(u_{2}\right)\right|$ $\leq\left|u_{1}-u_{2}\right|$. In the case when $u_{1} \leq t \leq u_{2}$, we have

$$
\begin{aligned}
& \left|(T(t) x)\left(u_{1}\right)-(T(t) x)\left(u_{2}\right)\right| \\
& \quad \leq\left|(T(t) x)\left(u_{1}\right)-(T(t) x)(t)\right|+\left|(T(t) x)(t)-(T(t) x)\left(u_{2}\right)\right| \\
& \quad \leq\left|u_{1}-t\right|+\left|t-u_{2}\right|=\left|u_{1}-u_{2}\right| .
\end{aligned}
$$

Therefore we have shown that $T(t) x \in C$ for $t \in[0,1]$ and $x \in C$. By the definition of $\{T(t): t \geq 0\}$, we have $T(t) x \in C$ for all $t \in[0, \infty)$ and $x \in C$. We next show that $\{T(t)$ : $t \geq 0\}$ is a one-parameter nonexpansive semigroup on $C$.

(sg1) Fix $t \in[0,1]$, and $x, y \in C$. We will prove that

$$
|(T(t) x)(u)-(T(t) y)(u)| \leq\|x-y\| \quad \forall u \in \Omega .
$$


We have

$$
|(T(t) x)(-1)-(T(t) y)(-1)|=|x(-1)-y(-1)| \leq\|x-y\| .
$$

For $u \geq t$, we have

$$
|(T(t) x)(u)-(T(t) y)(u)|=|x(u-t)-y(u-t)| \leq\|x-y\| .
$$

Fix $u$ with $0 \leq u \leq t$. In the case when $1-\alpha_{x}(1-t+u) \leq x(0)-t+u$ and $1-\alpha_{y}(1-t+$ $u) \leq y(0)-t+u$, we have

$$
\begin{aligned}
|(T(t) x)(u)-(T(t) y)(u)| & =|(x(0)-t+u)-(y(0)-t+u)| \\
& =|x(0)-y(0)| \leq\|x-y\| .
\end{aligned}
$$

In the case when $1-\alpha_{x}(1-t+u) \leq x(0)-t+u$ and $1-\alpha_{y}(1-t+u)>y(0)-t+u$, we have

$$
(T(t) y)(u)=\min \left\{1-\alpha_{y}(1-t+u), y(0)+t-u\right\} \geq y(0)-t+u
$$

Hence,

$$
\begin{aligned}
(T(t) x)(u)-(T(t) y)(u) & \leq(x(0)-t+u)-(y(0)-t+u)=x(0)-y(0) \leq\|x-y\| \\
(T(t) y)(u)-(T(t) x)(u) & \leq\left(1-\alpha_{y}(1-t+u)\right)-\left(1-\alpha_{x}(1-t+u)\right) \\
& =\alpha_{x}(1-t+u)-\alpha_{y}(1-t+u) \leq\|x-y\|
\end{aligned}
$$

hold. Therefore (2.19) holds. Similarly we can prove (2.19) in the other cases. On the other hand, we have

$$
\begin{aligned}
\|T(t) x-T(t) y\| & \geq \sup \{|(T(t) x)(u)-(T(t) y)(u)|: u \in\{-1\} \cup[t, \infty)\} \\
& =\sup \{|x(u)-y(u)|: u \in \Omega\}=\|x-y\| .
\end{aligned}
$$

Hence we have shown that

$$
\|T(t) x-T(t) y\|=\|x-y\|
$$

for $t \in[0,1]$ and $x, y \in C$. So, by the definition of $\{T(t): t \geq 0\},(2.26)$ holds for all $t \in$ $[0, \infty)$ and $x, y \in C$.

(sg2) It is clear that $T(0)$ is the identity mapping on $C$.

$(\operatorname{sg} 3)$ Fix $t_{1}, t_{2} \in[0,1 / 2]$ and $x \in C$. We will prove that

$$
\left(T\left(t_{1}\right) \circ T\left(t_{2}\right) x\right)(u)=\left(T\left(t_{1}+t_{2}\right) x\right)(u) \quad \forall u \in \Omega .
$$

We have

$$
\left(T\left(t_{1}\right) \circ T\left(t_{2}\right) x\right)(-1)=\left(T\left(t_{2}\right) x\right)(-1)=x(-1)=\left(T\left(t_{1}+t_{2}\right) x\right)(-1) .
$$


For $u \geq t_{2}$, we have

$$
\left(T\left(t_{1}+t_{2}\right) x\right)\left(t_{1}+u\right)=x\left(\left(t_{1}+u\right)-\left(t_{1}+t_{2}\right)\right)=x\left(u-t_{2}\right)=\left(T\left(t_{2}\right) x\right)(u) .
$$

For $u \in\left[0, t_{2}\right]$, since $t_{1}+u \leq t_{1}+t_{2}, 1-\alpha_{x}\left(1-t_{2}+u\right)=1-\alpha_{x}\left(1-\left(t_{1}+t_{2}\right)+\left(t_{1}+u\right)\right)$, $x(0)-t_{2}+u=x(0)-\left(t_{1}+t_{2}\right)+\left(t_{1}+u\right)$, and $x(0)+t_{2}-u=x(0)+\left(t_{1}+t_{2}\right)-\left(t_{1}+u\right)$, the two definitions of $\left(T\left(t_{1}+t_{2}\right) x\right)\left(t_{1}+u\right)$ and $\left(T\left(t_{2}\right) x\right)(u)$ coincide. Therefore

$$
\left(T\left(t_{1}+t_{2}\right) x\right)\left(t_{1}+u\right)=\left(T\left(t_{2}\right) x\right)(u) .
$$

So, for $u \geq t_{1}$,

$$
\begin{aligned}
\left(T\left(t_{1}\right) \circ T\left(t_{2}\right) x\right)(u) & =\left(T\left(t_{2}\right) x\right)\left(u-t_{1}\right) \\
& =\left(T\left(t_{1}+t_{2}\right) x\right)\left(t_{1}+\left(u-t_{1}\right)\right) \\
& =\left(T\left(t_{1}+t_{2}\right) x\right)(u) .
\end{aligned}
$$

Fix $u$ with $0 \leq u \leq t_{1}$. Then we have

$$
\begin{aligned}
1-\alpha_{T\left(t_{2}\right) x}\left(1-t_{1}+u\right) & =1-\sup \left\{\left(T\left(t_{2}\right) x\right)(s): s \in\{-1\} \cup\left[1-t_{1}+u, \infty\right)\right\} \\
& =1-\max \left\{x(-1), \sup \left\{x\left(s-t_{2}\right): s \in\left[1-t_{1}+u, \infty\right)\right\}\right\} \\
& =1-\alpha_{x}\left(1-t_{1}-t_{2}+u\right) .
\end{aligned}
$$

In the case when $1-\alpha_{T\left(t_{2}\right) x}\left(1-t_{1}+u\right)<\left(T\left(t_{2}\right) x\right)(0)-t_{1}+u$, we have

$$
\left(T\left(t_{1}\right) \circ T\left(t_{2}\right) x\right)(u)=\left(T\left(t_{2}\right) x\right)(0)-t_{1}+u .
$$

Since

$$
\begin{aligned}
1-\alpha_{x}\left(1-t_{1}-t_{2}+u\right) & =1-\alpha_{T\left(t_{2}\right) x}\left(1-t_{1}+u\right)<\left(T\left(t_{2}\right) x\right)(0)-t_{1}+u \\
& =\left(T\left(t_{1}+t_{2}\right) x\right)\left(t_{1}\right)-t_{1}+u,
\end{aligned}
$$

we have

$$
\begin{gathered}
\left(T\left(t_{1}+t_{2}\right) x\right)(u)=x(0)-t_{1}-t_{2}+u, \\
\left(T\left(t_{1}+t_{2}\right) x\right)\left(t_{1}\right)=x(0)-t_{1}-t_{2}+t_{1}=x(0)-t_{2}
\end{gathered}
$$

by Lemma 2.3. So,

$$
\begin{aligned}
\left(T\left(t_{1}\right) \circ T\left(t_{2}\right) x\right)(u) & =\left(T\left(t_{2}\right) x\right)(0)-t_{1}+u=\left(T\left(t_{1}+t_{2}\right) x\right)\left(t_{1}\right)-t_{1}+u \\
& =x(0)-t_{2}-t_{1}+u=\left(T\left(t_{1}+t_{2}\right) x\right)(u) .
\end{aligned}
$$

Similarly, we can prove that $\left(T\left(t_{1}\right) \circ T\left(t_{2}\right) x\right)(u)=\left(T\left(t_{1}+t_{2}\right) x\right)(u)$ in the cases when $1-$ $\alpha_{T\left(t_{2}\right) x}\left(1-t_{1}+u\right)>\left(T\left(t_{2}\right) x\right)(0)+t_{1}-u$ and $\left|1-\alpha_{T\left(t_{2}\right) x}\left(1-t_{1}+u\right)-\left(T\left(t_{2}\right) x\right)(0)\right| \leq t_{1}-$ $u$. Therefore $T\left(t_{1}\right) \circ T\left(t_{2}\right)=T\left(t_{1}+t_{2}\right)$. So, we have, for $t \in[1 / 2,1)$,

$$
T(t)=T\left(\frac{1}{2}\right) \circ T\left(t-\frac{1}{2}\right), \quad T(1)=T\left(\frac{1}{2}\right) \circ T\left(\frac{1}{2}\right) \circ T(0) .
$$


Fix $t_{1}, t_{2} \in[0, \infty)$. Then there exist $m_{1}, m_{2} \in \mathbb{N} \cup\{0\}$ and $t_{1}^{\prime}, t_{2}^{\prime} \in[0,1 / 2)$ satisfying $t_{1}=$ $m_{1} / 2+t_{1}^{\prime}$ and $t_{2}=m_{2} / 2+t_{2}^{\prime}$. We have

$$
\begin{aligned}
T\left(t_{1}\right) \circ T\left(t_{2}\right) & =T\left(\frac{1}{2}\right)^{m_{1}} \circ T\left(t_{1}^{\prime}\right) \circ T\left(\frac{1}{2}\right)^{m_{2}} \circ T\left(t_{2}^{\prime}\right)=T\left(\frac{1}{2}\right)^{m_{1}+m_{2}} \circ T\left(t_{1}^{\prime}\right) \circ T\left(t_{2}^{\prime}\right) \\
& =T\left(\frac{1}{2}\right)^{m_{1}+m_{2}} \circ T\left(\min \left\{t_{1}^{\prime}+t_{2}^{\prime}, \frac{1}{2}\right\}\right) \circ T\left(\max \left\{0, t_{1}^{\prime}+t_{2}^{\prime}-\frac{1}{2}\right\}\right) \\
& =T\left(t_{1}+t_{2}\right) .
\end{aligned}
$$

(sg4) For $x \in C$ and $t \in[0, \infty)$, we have

$$
\begin{aligned}
\|T(t) x-x\| & =\sup \{|(T(t) x)(u)-x(u)|: u \in[0, \infty)\} \\
& =\sup \{|(T(t) x)(u)-(T(t) x)(t+u)|: u \in[0, \infty)\} \\
& \leq \sup \{|u-(t+u)|: u \in[0, \infty)\}=t .
\end{aligned}
$$

Therefore we obtain

$$
\left\|T\left(t_{1}\right) x-T\left(t_{2}\right) x\right\|=\left\|T\left(\left|t_{1}-t_{2}\right|\right) x-x\right\| \leq\left|t_{1}-t_{2}\right|
$$

for $x \in C$ and $t_{1}, t_{2} \in[0,1]$. Therefore $T(\cdot) x$ is continuous for all $x \in C$.

We prove that

$$
\bigcap_{t \geq 0} F(T(t))=\left\{v_{s}: s \in\left[0, \frac{1}{2}\right]\right\} \cup\left\{w_{s}: s \in\left[0, \frac{1}{2}\right]\right\},
$$

where

$$
\begin{gathered}
v_{s}(u)= \begin{cases}1-s & \text { if } u=-1, \\
s & \text { if } u \in[0, \infty),\end{cases} \\
w_{s}(u)= \begin{cases}s & \text { if } u=-1, \\
\frac{1}{2} & \text { if } u \in[0, \infty) .\end{cases}
\end{gathered}
$$

Fix $s \in[0,1 / 2]$ and $t \in[0,1]$. Then we have

$$
\begin{gathered}
\left|1-\alpha_{v_{s}}(1-t+u)-v_{s}(0)\right|=|1-(1-s)-s|=0 \leq t-u, \\
\left|1-\alpha_{w_{s}}(1-t+u)-w_{s}(0)\right|=\left|1-\frac{1}{2}-\frac{1}{2}\right|=0 \leq t-u,
\end{gathered}
$$

for $u \in[0, t]$. So

$$
\begin{gathered}
\left(T(t) v_{s}\right)(u)=1-\alpha_{v_{s}}(1-t+u)=s=v_{s}(u) \\
\left(T(t) w_{s}\right)(u)=1-\alpha_{w_{s}}(1-t+u)=\frac{1}{2}=w_{s}(u)
\end{gathered}
$$


Hence, $T(t) v_{s}=v_{s}$ and $T(t) w_{s}=w_{s}$. Therefore, $v_{s}$ and $w_{s}$ are common fixed points of $\{T(t): t \geq 0\}$. Conversely, we assume that $x \in C$ is a common fixed point of $\{T(t): t \geq 0\}$. Put $s=x(0)$. Then we have

$$
x(t+u)=(T(t) x)(t+u)=x(t+u-t)=x(u)
$$

for all $u \in[0, \infty)$ and $t \in[0,1]$. So, $x(u)=x(0)=s$ hold for all $u \in[0, \infty)$. We also have

$$
s=x(0)=(T(1) x)(0)=1-\alpha_{x}(1-1+0)=1-\alpha_{x}(0)=\min \{1-x(-1), 1-s\} .
$$

Hence $x(-1) \leq 1-s$ and $s \leq 1 / 2$. If $s=1 / 2$, then $x=w_{x(-1)}$. If $s<1 / 2$, then $x(-1)=1-s$ and hence $x=v_{s}$. Therefore we have shown (2.41).

Define a function $f$ from $\mathbb{R}$ into $[0,1]$ by

$$
f(u)= \begin{cases}0, & \text { if } u \geq 0 \\ -u, & \text { if }-1 \leq u \leq 0 \\ u+2, & \text { if }-2 \leq u \leq-1 \\ 0, & \text { if } u \leq-2\end{cases}
$$

We finally show that

$$
(T(t) 0)(u)= \begin{cases}0, & \text { if } u=-1, \\ f(u-t), & \text { if } u \in[0, \infty) .\end{cases}
$$

Fix $t \in[0,1]$ and $u \in[0, t]$. Then we have

$$
1-\alpha_{0}(1-t+u)=1 \geq 0+t-u
$$

and hence

$$
(T(t) 0)(u)=0+t-u=t-u=f(u-t)
$$

because $-1 \leq u-t \leq 0$. Therefore

$$
(T(1) 0)(s)= \begin{cases}0, & \text { if } s=-1 \text { or } s \geq 1 \\ 1-s, & \text { if } 0 \leq s \leq 1\end{cases}
$$

Since

$$
\begin{aligned}
1-\alpha_{T(1) 0}(1-t+u) & =1-(1-(1-t+u))=1-t+u \\
& =(T(1) 0)(0)-t+u
\end{aligned}
$$

we have

$$
\begin{aligned}
(T(t+1) 0)(u) & =(T(t) \circ T(1) 0)(u)=(T(1) 0)(0)-t+u \\
& =1-t+u=f(u-(1+t)) .
\end{aligned}
$$


Therefore

$$
(T(2) 0)(s)= \begin{cases}0, & \text { if } s=-1 \text { or } s \geq 2 \\ 2-s, & \text { if } 1 \leq s \leq 2 \\ s, & \text { if } 0 \leq s \leq 1\end{cases}
$$

Since

$$
\left|1-\alpha_{T(2) 0}(1-t+u)-(T(2) 0)(0)\right|=|1-1-0|=0 \leq t-u,
$$

we have

$$
\begin{aligned}
(T(t+2) 0)(u) & =(T(t) \circ T(2) 0)(u) \\
& =1-\alpha_{T(2) 0}(1-t+u)=0=f(u-(2+t)) .
\end{aligned}
$$

Similarly, for $k \in \mathbb{N}$ with $k>2$, we can prove

$$
(T(t+k) 0)(u)=0=f(u-(k+t)) .
$$

Therefore we have shown (2.48). So, (2.5) clearly holds. This completes the proof.

\section{References}

[1] J. B. Baillon, Quelques propriétés de convergence asymptotique pour les semi-groupes de contractions impaires, C. R. Acad. Sci. Paris Sér. A-B 283 (1976), no. 3, A75-A78.

[2] J. B. Baillon and H. Brezis, Une remarque sur le comportement asymptotique des semigroupes non linéaires, Houston J. Math. 2 (1976), no. 1, 5-7 (French).

[3] L. P. Belluce and W. A. Kirk, Nonexpansive mappings and fixed-points in Banach spaces, Illinois J. Math. 11 (1967), 474-479.

[4] F. E. Browder, Nonexpansive nonlinear operators in a Banach space, Proc. Natl. Acad. Sci. USA 54 (1965), 1041-1044.

[5] R. E. Bruck, Jr., A common fixed point theorem for a commuting family of nonexpansive mappings, Pacific J. Math. 53 (1974), 59-71.

[6] R. DeMarr, Common fixed points for commuting contraction mappings, Pacific J. Math. 13 (1963), 1139-1141.

[7] K. Goebel and W. A. Kirk, Topics in Metric Fixed Point Theory, Cambridge Studies in Advanced Mathematics, vol. 28, Cambridge University Press, Cambridge, 1990.

[8] J.-P. Gossez and E. Lami Dozo, Some geometric properties related to the fixed point theory for nonexpansive mappings, Pacific J. Math. 40 (1972), 565-573.

[9] N. Hirano, Nonlinear ergodic theorems and weak convergence theorems, J. Math. Soc. Japan 34 (1982), no. 1, 35-46.

[10] T. C. Lim, A fixed point theorem for families on nonexpansive mappings, Pacific J. Math. 53 (1974), 487-493.

[11] I. Miyadera and K. Kobayasi, On the asymptotic behaviour of almost-orbits of nonlinear contraction semigroups in Banach spaces, Nonlinear Anal. 6 (1982), no. 4, 349-365.

[12] Z. Opial, Weak convergence of the sequence of successive approximations for nonexpansive mappings, Bull. Amer. Math. Soc. 73 (1967), 591-597.

[13] T. Suzuki, On strong convergence to common fixed points of nonexpansive semigroups in Hilbert spaces, Proc. Amer. Math. Soc. 131 (2003), no. 7, 2133-2136. 
[14] Some remarks on the set of common fixed points of one-parameter semigroups of nonexpansive mappings in Banach spaces with the Opial property, Nonlinear Anal. 58 (2004), no. 3-4, 441-458.

[15] T. Suzuki and W. Takahashi, Strong convergence of Mann's type sequences for one-parameter nonexpansive semigroups in general Banach spaces, J. Nonlinear Convex Anal. 5 (2004), no. 2, 209-216.

[16] W. Takahashi, Nonlinear Functional Analysis, Yokohama Publishers, Yokohama, 2000.

Tomonari Suzuki: Department of Mathematics, Kyushu Institute of Technology, 1-1 Sensui-cho, Tobata-ku, Kitakyushu 804-8550, Japan

E-mail address: suzuki-t@mns.kyutech.ac.jp 


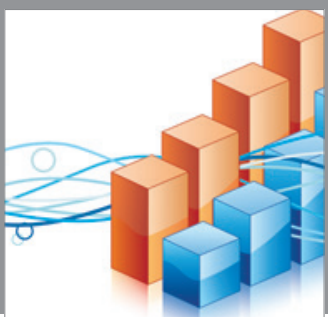

Advances in

Operations Research

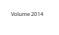

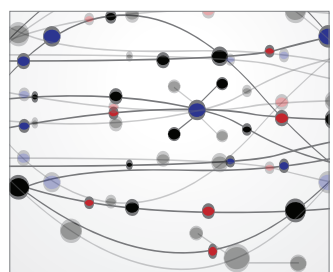

\section{The Scientific} World Journal
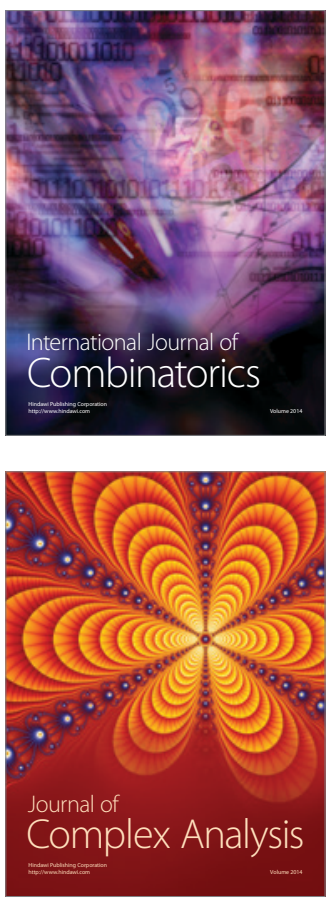

International Journal of

Mathematics and

Mathematical

Sciences
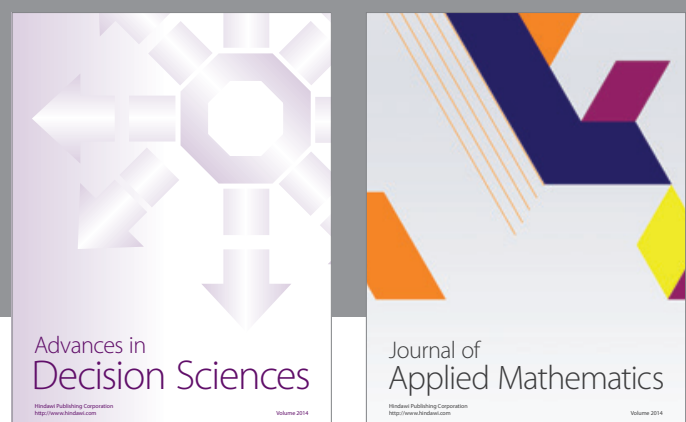

Journal of

Applied Mathematics
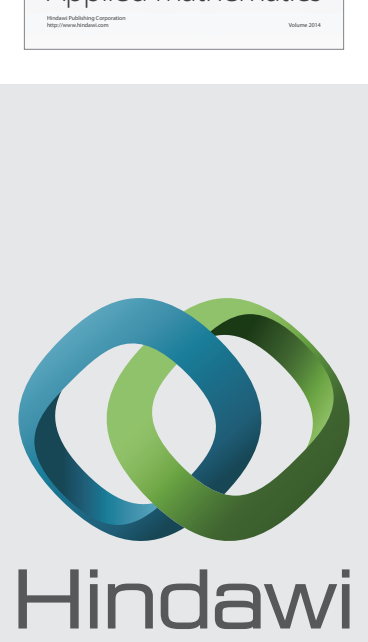

Submit your manuscripts at http://www.hindawi.com
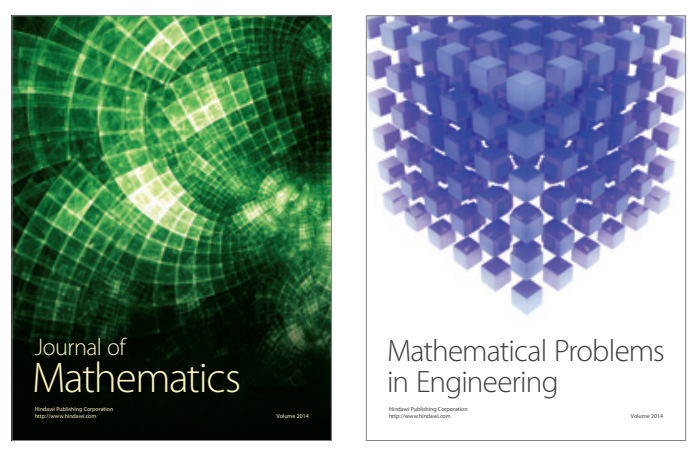

Mathematical Problems in Engineering
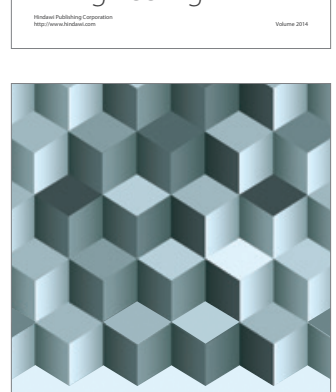

Journal of

Function Spaces
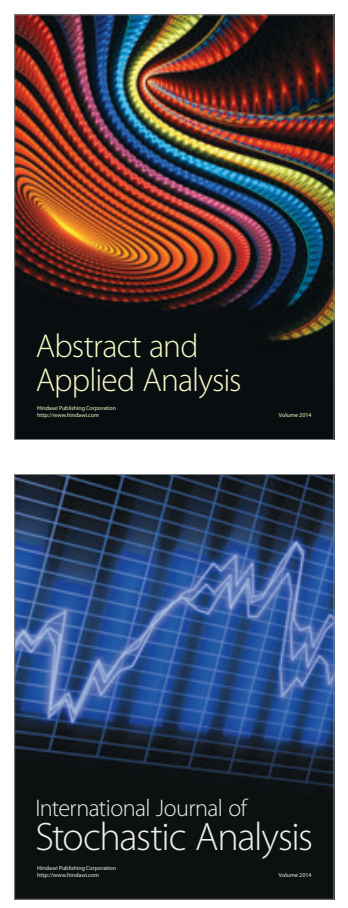

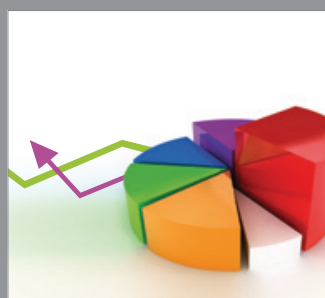

ournal of

Probability and Statistics

Promensencen
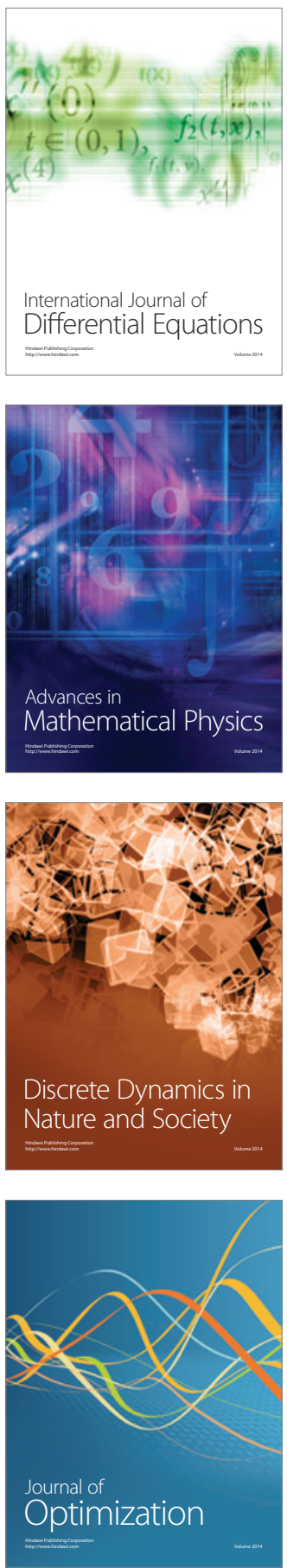